\title{
A Note on the Notes
}

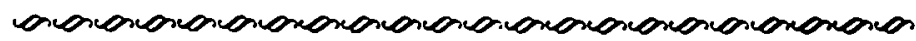

Notes of reference are printed at the end of the volume, following the list of "Texts and References." Notes of substance (with abridged references as needed) are printed in the text. It has not been found practicable to make this distinction quite absolute, but the reader with no interest in the source of information or quotation may be assured of losing little or nothing by ignoring the backnotes. 